\title{
Streamline Visualization of Diffusion Tensor Data based on Triangle Strips
}

\author{
Dorit Merhof $^{1,2}$, Markus Sonntag ${ }^{1,2}$, Frank Enders $^{1,2}$, \\ Christopher Nimsky ${ }^{2,3}$, Peter Hastreiter ${ }^{1,2}$, Günther Greiner ${ }^{1}$ \\ ${ }^{1}$ Computer Graphics Group, University of Erlangen-Nuremberg, Germany \\ ${ }^{2}$ Neurocenter, Dept. of Neurosurgery, University of Erlangen-Nuremberg, Germany \\ ${ }^{3}$ Dept. of Neurosurgery, University of Erlangen-Nuremberg, Germany \\ Email: dorit.merhof@informatik.uni-erlangen.de
}

\begin{abstract}
For the visualization of diffusion tensor imaging data, different approaches have been presented such as scalar metrics, glyphs or streamlines. Thereby, streamline techniques commonly referred to as fiber tracking provide a comprehensive and intuitive representation. For this reason, they are preferably applied for preoperative planning. The visualization of streamlines is solved by rendering lines or tubes to achieve even more significant results. However, the number of streamlines for a tracking of the whole brain or very dense tract systems may be immense, making a mesh-based tube visualization inefficient. To overcome this problem, we developed an alternative visualization technique for tubes by using textured triangle strips.
\end{abstract}

\section{Introduction}

Diffusion tensor imaging (DTI) is a magnetic resonance acquisition technique which has emerged in recent years providing valuable information about the location of white matter tracts within the human brain in vivo. DTI measures diffusion of water which is anisotropic in tissue with high degree of directional organization such as white matter. Thereby, information about the location of neuronal fibers is provided which is of great interest for neurosurgery.

The reconstruction of white matter tracts from DTI data is commonly solved by tracking algorithms which utilize streamline techniques known from flow visualization. This visualization strategy provides an intuitive understanding of the spatial relation between fibers and space occupying lesions and is therefore a valuable supplement for neurosurgical planning [1,2].

Almost all approaches presented in the context of fiber tracking focus on strategies to increase the precision of the computed streamlines. Thereby, filter approaches [3] have been presented as well as alternative streamline propagation techniques [4]. Recent approaches consider wavefront evolution techniques [5] as well as accurate treatment of crossing and branching fibers [6]. However, for the appropriate visualization of the derived fibers, only standard techniques such as lines or tubes are used in most cases.

Currently, most tools for DTI processing use lines or tubes to render fibers. However, utilizing simple line rendering has several disadvantages. In case of curved fibers, 
lines wider than one pixel suffer from gaps between neighboring segments. Furthermore, a constant width independent of the distance to the camera restricts the quality of visualization. Tubes on the other hand enable a significantly improved visualization, but rendering becomes much more time consuming due to the high geometric complexity restricting their interactive application. To overcome these limitations, we propose an alternative visualization technique using textured triangle strips which are reoriented depending on the viewing direction. Thereby, a visualization very similar to tubes is obtained but with a significantly reduced number of triangles enabling interactive rendering.

\section{Material}

All datasets used in this work were measured using a Siemens MR Magnetom Sonata Maestro Class 1.5 Tesla scanner. The specifications of the gradient system were a field strength of up to $40 \mathrm{mT} / \mathrm{m}$ (effective $69 \mathrm{mT} / \mathrm{m}$ ) and a slew rate of up to $200 \mathrm{~T} / \mathrm{m} / \mathrm{s}$ (effective $346 \mathrm{~T} / \mathrm{m} / \mathrm{s}$ ) for clinical application.

DTI datasets were acquired using a field of view of $240 \mathrm{~mm}$ resulting in a voxel size of $1.875 \times 1.875 \times 1.9 \mathrm{~mm}^{3}$. For each of the six diffusion weighted datasets (gradient directions $( \pm 1,1,0),( \pm 1,0,1)$ and $(0,1, \pm 1))$ and the reference dataset, sixty slices with no intersection gap and an acquisition matrix of $128 \times 128$ pixels were measured.

\section{Methods}

In a first step, fibers were computed using a fiber tracking approach incorporating trilinear tensor interpolation and fourth order Runge-Kutta integration. Fractional anisotropy was used as termination threshold for tracking. Optionally, oriented filters [3] were applied for noise reduction.

Subsequently, triangle strips were reconstructed by creating two triangles for each fiber segment. Thereby, a pair of triangle vertices $\boldsymbol{P}_{i} \pm 1 / 2 \cdot \boldsymbol{d}_{i}$ was generated from the original streamline vertex $\boldsymbol{P}_{i}$ using a displacement vector $\boldsymbol{d}_{i}$ resulting from the cross product of viewing direction $\boldsymbol{v}_{i}$ and streamline tangent $\boldsymbol{t}_{i}$. The displacement vector was normalized and scaled to obtain a user-defined width of the triangle strip:

$$
\boldsymbol{d}_{i}=c_{w i d t h} \cdot \frac{\boldsymbol{v}_{i} \times \boldsymbol{t}_{i}}{\left\|\boldsymbol{v}_{i} \times \boldsymbol{t}_{i}\right\|} .
$$

Regarding coloring of the triangle strips, a primary color was determined according to the standard color scheme commonly used in DTI visualizations [7]. Thereby, the normalized principal eigenvector components of the local trilinearly interpolated tensor were used as RGB values. To provide a 3D tube effect, texturing of the triangles became darker when approaching the boarder of the triangle strip. This was achieved by using a $1 \mathrm{D}$ texture with a luminance and an alpha channel. The luminance channel of the texture was used to store the ambient illumination, the alpha channel stored the specular term. A sine function was used to darken the texture when approaching the border to obtain the visual effect of an illuminated tube. In case of the alpha channel, the result of the sine function was raised to a power of 16 to generate specular highlights. 
To obtain a realistic representation at the end of each fiber, tapering was used. Thereby, two further triangles were generated for each end of the fiber to terminate the streamline. Tapering provides a more tube-like representation at the end of each fiber where otherwise the flat geometry would become apparent.

\section{Results and Discussion}

In comparison to lines, tubes provide a much better 3D representation of streamlines. Rendering of lines results in gaps between neighboring segments if the lines are wider than one pixel. More severely, lines have a constant width independent of the distance to the camera which highly restricts the quality of visualization. Tubes on the other hand enable a significantly improved visualization, but rendering becomes much more time consuming due to the high geometric complexity. Triangle strips provide a good tradeoff between visual quality and interactive application. A comparison between rendered lines, geometry tubes and triangle strips simulating tubes is presented in Figure 1.
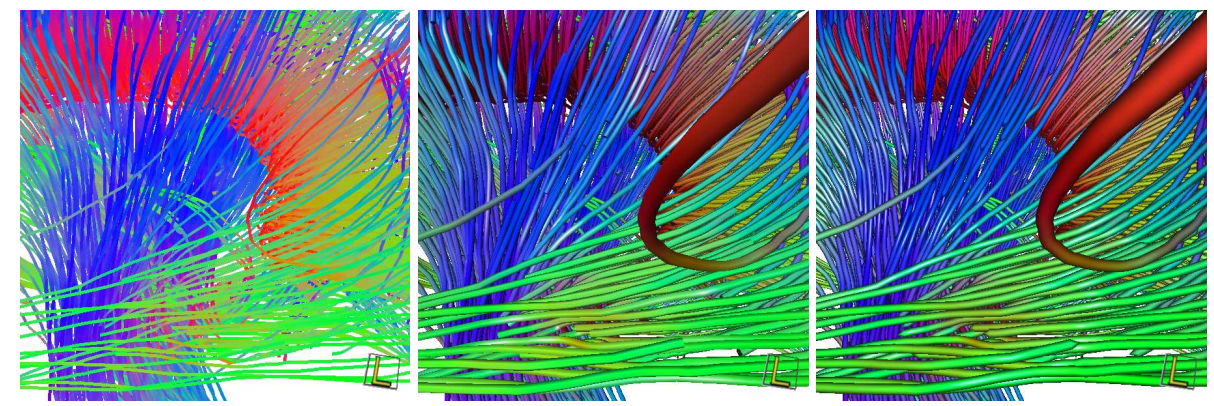

Fig. 1. Comparison of standard fiber rendering using lines (left) or tubes modeled with a triangle mesh (middle) and an alternative triangle-strip based tube representation (right).

The approach was evaluated for whole brain trackings in brain tumor patients as well as healthy volunteers based on a data basis consisting of 28 datasets. Depending on the density of seed points and the tracking threshold, a tracking of the whole brain comprised about 1000 up to 10000 fibers. The average number of points for each fiber was in the range of 150 . According to our expectations, rendering of tubes was by far slower than our novel technique due to a much higher number of triangles (Table 1). In our approach, two triangles are needed for each fiber segment. Contrary to that the geometric representation of a tube segment requires at least 12 or more triangles to obtain a sufficient rendering result. In comparison to the rendering of lines, our approach achieved lower frame rates but the visualization was considerably improved.

The performance measurements were obtained for a rendering viewport of $600 \times 800$ pixels on a NVidia Geforce 6800 graphics card with $256 \mathrm{MB}$ graphics memory. A tracking of the whole brain consisting of approximately 300000 vertices was generated and used for the measurements. Table 1 shows the measurement results for lines, 
geometry-based tubes with 12 triangles per segment and triangle strips. In case of tubes, 3.6 million triangles were rendered. In case of triangle strips 0.6 million triangles were necessary. Variations of the frame rate also originate from implementation aspects depending on whether Vertex Buffer Objects (VBOs), Vertex Arrays (VAs), Display Lists (DLs) or Immediate Mode (IM) were applied.

\begin{tabular}{lrrrr}
\hline & VBOs & VAs & DLs & IM \\
\hline Lines (no illumination) & $242.5 \mathrm{fps}$ & $135.4 \mathrm{fps}$ & $219.9 \mathrm{fps}$ & $45.3 \mathrm{fps}$ \\
Lines (illuminated) & $153.3 \mathrm{fps}$ & $135.1 \mathrm{fps}$ & $153.1 \mathrm{fps}$ & $45.2 \mathrm{fps}$ \\
Tubes (no illumination) & $10.0 \mathrm{fps}$ & $9.9 \mathrm{fps}$ & $8.9 \mathrm{fps}$ & $2.7 \mathrm{fps}$ \\
Tubes (illuminated) & $10.0 \mathrm{fps}$ & $9.9 \mathrm{fps}$ & $8.9 \mathrm{fps}$ & $2.7 \mathrm{fps}$ \\
Triangle Strips Tapering (no illumination) & $80.9 \mathrm{fps}$ & $39.7 \mathrm{fps}$ & $81.2 \mathrm{fps}$ & $14.6 \mathrm{fps}$ \\
Triangle Strips Tapering (illuminated) & $61.9 \mathrm{fps}$ & $39.7 \mathrm{fps}$ & $61.8 \mathrm{fps}$ & $14.6 \mathrm{fps}$ \\
Triangle Strips (no illumination) & $85.3 \mathrm{fps}$ & $42.2 \mathrm{fps}$ & $85.6 \mathrm{fps}$ & $16.6 \mathrm{fps}$ \\
Triangle Strips (illuminated) & $65.4 \mathrm{fps}$ & $42.2 \mathrm{fps}$ & $65.1 \mathrm{fps}$ & $16.6 \mathrm{fps}$ \\
\hline
\end{tabular}

Table 1. Frame rates in fps (frames per second) depending on the visualization technique (lines, tubes or triangle strips) and illumination for different graphics card storage options.

With respect to clinical application, tube representations enable a better inspection of the data (Figure 2) and consequently an improved diagnosis. In the context of practical use, interactivity of the rendering procedure is of major concern since clinical acceptance is directly related to usability and interactive manipulation. The presented approach provides the physician with interactive high quality tube renderings of tracked fibers.

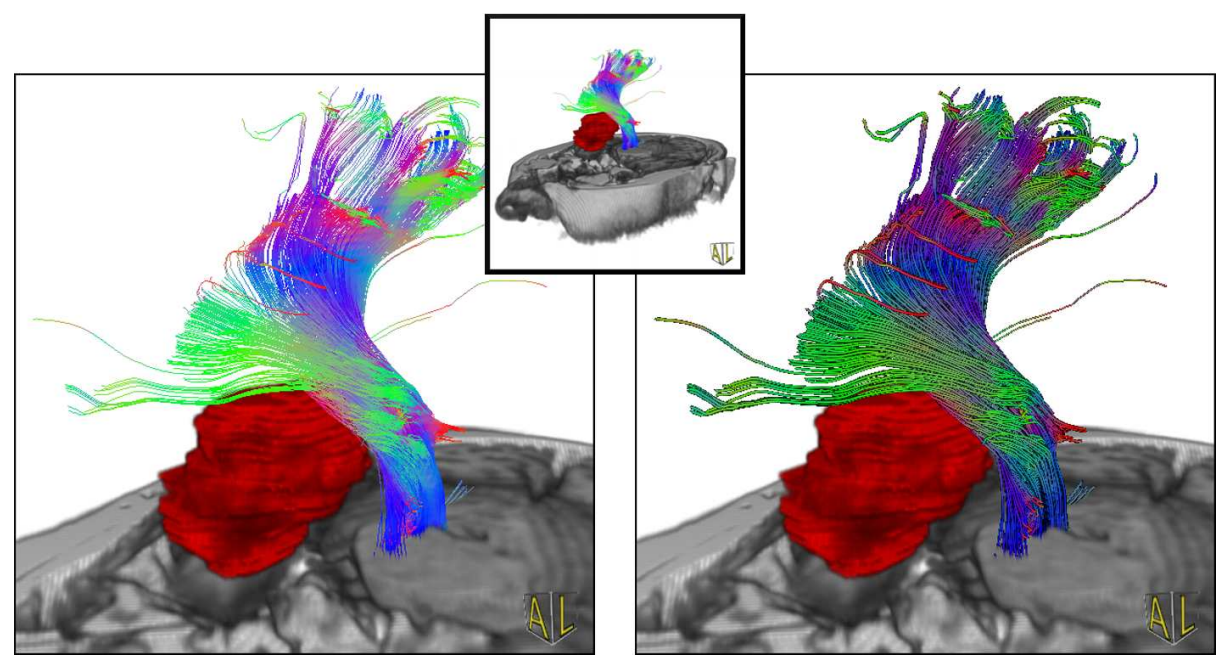

Fig. 2. Comparison of line rendering (left) and triangle-strip based tube representation (right) in a 68-year old male patient with an astrocytom (WHO grade III) adjacent to the pyramidal tract. 


\section{Conclusion}

We presented a novel method for the visualization of fiber tracts derived from DTI data using textured triangle strips which is an alternative visualization strategy for tubes. This approach overcomes the disadvantages of both line and geometry-based tube representation. It is more efficient in terms of rendering times compared to tubes which is of particular importance if a large number of streamlines has to be visualized. In comparison to lines, the tube visualization provided by triangle strips is much better. Overall, the presented technique clearly improves the visualization compared to line representations and circumvents high rendering times encountered with tubes. For medical application, this is of high value since an interactive visualization is provided showing tube-like, high quality fibers.

\section{Acknowledgments}

This work was supported by the Deutsche Forschungsgemeinschaft in the context of SFB 603, Project C9 and the Graduate Research Center "3D Image Analysis and Synthesis".

\section{References}

1. Clark, C., Barrick, T., Murphy, M., Bell, B.: White matter fiber tracking in patients with spaceoccupying lesions of the brain: a new technique for neurosurgical planning? Neuroimage 20 (2003) 1601-1608

2. Nimsky, C., Ganslandt, O., Hastreiter, P., Wang, R., Benner, T., Sorensen, A., Fahlbusch, R.: Intraoperative diffusion-tensor MR imaging: shifting of white matter tracts during neurosurgical procedures - initial experience. Radiology 234 (2005) 218-225

3. Zhukov, L., Barr, A.: Oriented Tensor Reconstruction: Tracing Neural Pathways from Diffusion Tensor MRI. In: Proc. IEEE Visualization. (2002)

4. Lazar, M., Weinstein, D., Tsuruda, J., Hasan, K., Arfanakis, K., Meyerand, M., Badie, B., Rowley, H., Haughton, V., Field, A., Alexander, A.: White matter tractography using diffusion tensor deflection. Human Brain Mapping 18 (2003) 306-321

5. Jackowski, M., Kao, C., Qiu, M., Constable, R., Staib, L.: White matter tractography by anisotropic wavefront evolution and diffusion tensor imaging. Medical Image Analysis 9 (2005) 427-440

6. Vilanova, A., Berenschot, G., van Pul, C.: Dti visualization with streamsurfaces and evenlyspaced volume seeding. In: Proc. Joint EG/IEEE TCVG VisSym. (2004) 173-182

7. Pajevic, S., Pierpaoli, C.: Color schemes to represent the orientation of anisotropic tissues from diffusion tensor data: Application to white matter fiber tract mapping in the human brain. Magnetic Resonance in Medicine 42 (1999) 526-540 